\title{
Desempenho Produtivo de Cabritos Submetidos a Diferentes Períodos de Aleitamento ${ }^{1}$
}

\author{
Jorge Luiz de Farias Ramos ${ }^{2}$, Roberto Germano Costa ${ }^{3}$, Ariosvaldo Nunes de Medeiros ${ }^{4}$
}

\begin{abstract}
RESUMO - O experimento foi realizado com o objetivo de avaliar desempenho produtivo de cabritos submetidos a diferentes períodos de aleitamento. Foram utilizados 42 caprinos mestiços de raça Alpina, sendo 21 fêmeas e 21 machos inteiros, distribuídos em três tratamentos com 14 animais: T1- desmame aos 56, T2 - desmame aos 70 e T3 - desmame aos 84 dias de idade. A dieta líquida, até os 35 dias de idade, foi leite de cabra, fornecido em duas mamadas e, após este período, passou a ser fornecida apenas em uma mamada, à tarde. O delineamento experimental utilizado foi inteiramente casualisado, com sete repetições, em esquema fatorial 3x2, com os fatores época de desmame e sexo (macho e fêmea). As médias de pesos, para os tratamentos, aos 182 dias de idade foram: $\mathrm{T} 1=19,3^{\mathrm{b}}, \mathrm{T} 2=20,3^{\mathrm{ab}}$ e T3=21,4 $\mathrm{kg}$. No período de 85 aos 182 dias de idade, não se verificou diferença significativa no ganho de peso entre os tratamentos, cujas médias foram: $\mathrm{T} 1=65^{\mathrm{a}}$, $\mathrm{T} 2=57^{\mathrm{a}} \mathrm{e} \mathrm{T} 3=66^{\mathrm{a}} \mathrm{g} /$ dia. $\mathrm{O}$ desaleitamento aos 56 dias de idade proporcionou desenvolvimento satisfatório dos cabritos.
\end{abstract}

Palavras-chave: caprino, desmame, ganho de peso

\section{Productive Performance of Goats Submitted to Different Periods of Suckling}

ABSTRACT - The research was carried out to evaluate the performance of kids submitting of different periods of suckling. Forty-two Alpine goats crossbred (21 males and 21 females) were assigned to three treatments with 14 replicates: T1 weaning at 56; T2 weaning at the 70 and T3 weaning at the 84 days old. The liquid diet (goat milk) was fed up to 35 days old, in two suckling and, after, the animals received the same quantity of goat milk at one suckling, in the afternoon. An experimental randomized completely design in 3 x 2 factorial scheme (three weaning period x two sex), with seven replicates, was used. Average weight for the respective treatments at 182 days old were: $\mathrm{T} 1=19.3^{\mathrm{b}} ; \mathrm{T} 2=20.3^{\mathrm{ab}}$ and $\mathrm{T} 3=21.4^{\mathrm{a}} \mathrm{kg}$. No significant differences among the treatments were observed for weight gain, from 85 to 182 days old, with means of T1 =65 $;$; T2 $=57^{\mathrm{a}}$ and T3 $=66^{\mathrm{a}} \mathrm{g} / \mathrm{day}$. Weaning at 56 days showed a satisfactory goat development.

Key Words: goat, weight gain, weaning

\section{Introdução}

A exploração de caprinos tem buscado a introdução de novas técnicas de manejo e melhoramento, visando sua consolidação como pecuária racional para o semi-árido, representando importante fonte de proteína animal e atendendo parte das necessidades nutricionais das populações carentes do nordeste brasileiro. Para o aumento da produtividade desses pequenos ruminantes, há a necessidade de incorporar ao sistema produtivo regional técnicas adequadas que viabilizem o desenvolvimento dessa atividade.

O aleitamento artificial vem sendo adotado com sucesso na atividade caprina como um dos procedimentos utilizados para elevar a lucratividade da exploração. Isto possibilita colocar no mercado maior quantidade de leite e derivados, visando atender à demanda crescente por produtos de origem caprina.

A prática do aleitamento artificial na caprinocultura leiteira é comum e seus efeitos sobre o desempenho dos animais e a eficiência deste sistema estão relacionados a vários fatores, a saber: ingestão do colostro nas primeiras horas após o nascimento, fases de aleitamento, freqüência das mamadas, temperatura do leite, quantidade de leite oferecida e dieta sólida de boa qualidade, fornecida a partir da segunda semana de vida. Quando o aleitamento artificial é utilizado, recomenda-se que as crias sejam separadas das mães logo após o nascimento, sendo o colostro fornecido em mamadeira, caixa de aleitamento ou balde (Mouchrek \& Moulin, 1987).

\footnotetext{
${ }^{1}$ Parte da Dissertação de Mestrado em Zootecnia apresentada ao CCA/UFPB.

${ }^{2}$ Pesquisador da EMEPA - PB. João Pessoa-PB. E.mail: jlfcabras@ig.com.br

3 Professor do Departamento de Agropecuária, do Centro de Formação de Tecnólogos, Campus III da UFPB - CEP: 58.220-000 Bananeiras-PB. E.mail: rgermano@cft.ufpb.br

${ }^{4}$ Professor do Departamento de Zootecnia, do Centro de Ciências Agrárias, Campus III da UFPB - CEP: 58.397-000 - Areia - PB. E.mail: medeiros@cca.ufpb.br
} 
Existem diversos sistemas de aleitamento artificial para cabritos, ocorrendo variações no tipo, na quantidade, freqüência e no período de fornecimento da dieta líquida (Mouchrek, 1991). Esses períodos de fornecimento do leite variam de 35 a 90 dias, estando relacionados à oferta e à qualidade dos alimentos sólidos e o conseqüente desempenho dos cabritos.

Medeiros (1996) comenta que, ao longo dos anos, muitos pesquisadores vêm tentando reduzir a quantidade de leite oferecida aos cabritos, bem como as fases de aleitamento, sem provocar prejuízos ao desenvolvimento dos animais tornando o manejo mais simples e econômico.

A precocidade no fornecimento da dieta sólida para cabritos é importante tanto para seu desempenho, como para o desenvolvimento do rúmen, tornando-o ruminante mais cedo (Susin, 1990). Cabritos submetidos à dieta de leite prolongada podem sofrer retardamento no desenvolvimento do rúmen, tornando o sistema produtivo oneroso, visto que, como ruminante, o caprino pode utilizar alimentos menos nobres que o leite e obter desenvolvimento satisfatório (Louca et al., 1975; Owen \& Paiva, 1980).

Nesse contexto, ressalta-se a importância do desaleitamento precoce, visando incrementar a produção de carne caprina a custos reduzidos para o produtor. Este trabalho foi conduzido com o objetivo de avaliar o desempenho produtivo de cabritos submetidos a diferentes períodos de aleitamento.

\section{Material e Métodos}

O experimento foi realizado no Setor de Caprinocultura do Centro de Formação de Tecnólogos, Campus IV da Universidade Federal da Paraíba.
Foram utilizados 42 caprinos mestiços da raça Alpina, sendo 21 fêmeas e 21 machos inteiros, distribuídos em três tratamentos, cada um com 14 animais:

Tratamento 1 (T1) = desmame aos 56 dias de idade; Tratamento 2 (T2) = desmame aos 70 dias de idade; Tratamento $3(\mathrm{~T} 3)$ = desmame aos 84 dias de idade

O leite era fornecido aos cabritos logo após a ordenha. As quantidades e freqüência do aleitamento encontram-se na Tabela 1.

Os cabritos foram aleitados em mamadeiras individuais, com controle da ingestão, de acordo com as fases pré-estabelecidas, com a dieta oferecida às 7 e 16 h. Após o aleitamento, as mamadeiras foram lavadas e desinfectadas.

Durante a fase experimental, os cabritos foram alimentados com capim-elefante, da variedade Cameroon (Pennisetum purpureum Schun), em forma de feno triturado em partículas de $3 \mathrm{~cm}$, apresentando 9\% de proteína bruta, e ração concentrada do tipo comercial, com $14 \%$ de proteína bruta.

$\mathrm{O}$ volumoso e o concentrado foram fornecidos aos animais partir dos sete dias de vida, com o consumo de alimentos avaliado por grupo. O volumoso foi fornecido ad libitum, no início da manhã e no final da tarde, enquanto o concentrado foi fornecido ad libitum, até $13^{\mathrm{a}}$ semana e, após essa fase, limitou-se o consumo em $400 \mathrm{~g} / \mathrm{animal} / \mathrm{dia}$.

A água foi fornecida ad libitum, em baldes plásticos, e renovada duas vezes ao dia. As sobras do volumoso e do concentrado foram previamente retiradas e pesadas, como forma de medir o consumo diário por grupo. A cada 15 dias, foram coletadas amostras para avaliação da composição química do alimento.

Os cabritos foram pesados logo após o nascimento e a cada sete dias, até alcançarem 182 dias de vida. As pesagens foram realizadas, pela manhã, antes do

Tabela 1 - Fornecimento da dieta líquida aos animais experimentais Table 1 - Liquid diet fed to experimental animals

\begin{tabular}{|c|c|c|c|c|}
\hline \multirow[t]{2}{*}{$\begin{array}{l}\text { Idade (dias) } \\
\text { Age (days) }\end{array}$} & \multirow[t]{2}{*}{$\begin{array}{l}\text { Tipo de aleitamento } \\
\text { Suckling type }\end{array}$} & \multicolumn{3}{|c|}{$\begin{array}{c}\text { Quantidade/animal/dia } \\
\text { Amount/animal/day }\end{array}$} \\
\hline & & $\begin{array}{l}\text { Manhã } \\
\text { Morning }\end{array}$ & $\begin{array}{c}\text { Tarde } \\
\text { Afternoon }\end{array}$ & $\begin{array}{l}\text { Total } \\
\text { Total }\end{array}$ \\
\hline $0-3$ & Colostro & & & \\
\hline & Colostrum & Ad libitum & Ad libitum & - \\
\hline $4-35$ & $\begin{array}{l}\text { Leite de cabra } \\
\text { Goat milk }\end{array}$ & 0,5 & 0,5 & 1,0 \\
\hline $36-84$ & $\begin{array}{l}\text { Leite de cabra } \\
\text { Goat milk }\end{array}$ & - & 1,0 & 1,0 \\
\hline
\end{tabular}

R. Bras. Zootec., v.33, n.3, p.684-690, 2004 
fornecimento da alimentação, em balança com precisão de 100 gramas.

O desempenho corporal dos animais foi analisado através do peso vivo e do ganho em peso, durante cinco fases distintas: a) do nascimento aos 56 dias de vida; b) dos 57 aos 70 dias; c) dos 71 aos 84 dias; d) dos 85 aos 182 dias de vida e e) do nascimento aos 182 dias de vida.

O delineamento experimental utilizado foi inteiramente casualisado com sete repetições, em esquema fatorial $3 \times 2$, com os fatores época de desmame e sexo. As médias obtidas dos tratamentos foram comparadas pelo o teste Duncan, a 5\% de probabilidade. O modelo contemplou a interação tratamento $\mathrm{x}$ sexo, que não apresentou efeito significativo. Os dados obtidos foram analisados utilizando-se o programa Statistical Analysis System (SAS, 1992).

\section{Resultados e Discussão}

\section{Consumo de alimentos}

O consumo médio diário de matéria seca (MS) do concentrado e do volumoso fornecidos aos cabritos encontra-se na Tabela 2. Esse consumo, em função da idade do animal, em dias, pode ser estimado pela equação: $Y=-117,81+6,987 x-0,181 x^{2}\left(R^{2}=0,97\right)$.

Considerando o consumo de matéria seca da dieta sólida, observou-se uma relação concentrado:volumoso (C:V), entre os 14 e 82 dias de idade, de 88,9:11,1 (T1); 88,9:11,1 (T2) e 90,8:9,2 (T3). A partir dessa idade, até os 182 dias, os animais de todos os tratamentos já se encontravam consumindo todo o concentrado pré-estabelecido (400 g), ocorrendo elevação no consumo de volumoso, resultando em uma relação C:V de: 71,6:28,4 (T1); 68,6:31,4 (T2) e 67,9:32,1 (T3).

Os consumos de MS, em relação ao peso vivo, foram: $\mathrm{T} 1=2,9 ; \mathrm{T} 2=3,0$ e T3=2,7\%, que são condizentes com os valores de 2,5 a 3,0\%, estabelecidos para animais em crescimento (NRC, 1981).

Os cabritos aos 56 dias de idade apresentaram ingestão média diária de 227,7 g MS, resultado próximo àquele estabelecido pelo NRC (1981), de $240 \mathrm{~g}$ MS/dia para $10 \mathrm{~kg}$ de peso vivo.

O concentrado e o volumoso consumidos propiciaram ingestão média diária de PB de 31,8 g/animal/dia, valor suficiente para atender às exigências de mantença dos cabritos com peso vivo $10 \mathrm{~kg}$, uma vez que o NRC (1981) recomenda, para cabritos criados em sistemas intensivos, consumo de proteína bruta de 27 g/animal/dia. Esses resultados são semelhantes aos relatados por Medeiros (1996), trabalhando com o mesmo rebanho.

Desenvolvimento ponderal de cabritos durante o aleitamento

Consta na Tabela 3 o desempenho médio dos

Tabela 2 - Consumo médio diário de matéria seca (MS) do concentrado (C) e volumoso (V) durante cada semana (g/animal/dia)

Table 2 - Average daily dry matter intake(MS) of the concentrate (C) and roughage (V) during each week (g/animal)

\begin{tabular}{|c|c|c|c|c|c|c|c|}
\hline \multirow{3}{*}{$\begin{array}{l}\text { Idade (Dias) } \\
\text { Age (Day) }\end{array}$} & \multicolumn{6}{|c|}{$\begin{array}{c}\text { Tratamentos } \\
\text { Treatments }\end{array}$} & \multirow{3}{*}{$\begin{array}{c}\text { Médias } \\
\text { Means } \\
\mathrm{C}+\mathrm{V}\end{array}$} \\
\hline & \multicolumn{2}{|c|}{$\mathrm{T} 1$} & \multicolumn{2}{|c|}{$\mathrm{T} 2$} & \multicolumn{2}{|c|}{ T3 } & \\
\hline & $\mathrm{C}$ & $\mathrm{V}$ & $\mathrm{C}$ & $\mathrm{V}$ & $\mathrm{C}$ & $\mathrm{V}$ & \\
\hline 14 & 7,3 & 5,8 & 8,2 & 5,1 & 7,7 & 4,2 & 12,7 \\
\hline 28 & 24,9 & 6,2 & 22,6 & 5,1 & 20,7 & 4,6 & 28,0 \\
\hline 42 & 84,1 & 13,9 & 106,9 & 15,8 & 113,4 & 10,5 & 114,9 \\
\hline 56 & 198,7 & 20,5 & 209,5 & 24,8 & 203,6 & 26,1 & 227,7 \\
\hline 70 & 324,5 & 34,9 & 313,2 & 25,7 & 325,4 & 28,6 & 350,7 \\
\hline 84 & 290,4 & 47,3 & 331,0 & 47,6 & 338,7 & 36,7 & 363,9 \\
\hline 98 & 338,7 & 72,3 & 338,7 & 88,0 & 338,7 & 97,0 & 424,5 \\
\hline 112 & 338,7 & 119,8 & 338,7 & 120,6 & 338,7 & 128,3 & 461,6 \\
\hline 126 & 338,7 & 114,5 & 338,7 & 107,6 & 338,7 & 125,1 & 454,4 \\
\hline 140 & 338,7 & 114,9 & 338,7 & 154,8 & 338,7 & 153,6 & 479,8 \\
\hline 154 & 338,7 & 132,0 & 338,7 & 135,3 & 338,7 & 176,0 & 486,5 \\
\hline 168 & 338,7 & 223,3 & 338,7 & 252,2 & 338,7 & 241,6 & 577,7 \\
\hline 182 & 338,7 & 221,7 & 338,7 & 264,4 & 338,7 & 230,0 & 577,4 \\
\hline
\end{tabular}

R. Bras. Zootec., v.33, n.3, p.684-690, 2004 
cabritos nas diferentes idades, durante as fases de aleitamento. Considerando-se o peso vivo dos cabritos como fator preponderante para se estabelecer o procedimento de desmame, observou-se que os animais, aos 56 dias de idade, apresentaram peso médio igual ou superior a $10 \mathrm{~kg}$, demonstrando a viabilidade da utilização do desaleitamento a partir desta fase da vida.

Neste trabalho, o esquema de aleitamento aos 56 dias de idade proporcionou desenvolvimento satisfatório aos animais. Nessa idade, os animais atingiram peso médio equivalente a 3,7 vezes o seu peso ao nascimento, superior a referência de 2,5 vezes estabelecida por Sanches (1985) e MorandFehr et al. (1982) como o ideal para realização do desmame, comprovando, assim, m bom desenvolvimento dos animais durante a fase de aleitamento.

Os resultados obtidos de peso médio para os cabritos, aos 56 dias de idade, foram semelhantes aos encontrados por Mouchreck \& Moulin (1987) e superiores aos relatados por Castro (1991), Ugiette (1993) e Medeiros (1996) e podem, nos três tratamentos, ser considerados satisfatórios para promoverem o desmame, associando-se ao fato de os animais estarem consumindo mais de $200 \mathrm{~g}$ de MS/dia.

Os animais desaleitados aos 70 e 84 dias de idade apresentaram, respectivamente, pesos médios de 13,0 e $14,9 \mathrm{~kg}$, resultados próximos aos encontrados por Susin (1990), que utilizou leite de cabra no aleitamento de cabritos de raças leiteiras, e de Barros et al. (1998), que, trabalhando com cabritos de raças leiteiras, utilizaram o leite de vaca.
Comparando-se o peso médio dos cabritos aos 84 dias, observou-se que os animais pertencentes aos tratamentos 2 e 3 apresentaram maior peso médio em relação aos animais do tratamento 1 , mostrando o efeito favorável de um período de aleitamento mais longo que os 56 dias do tratamento 1. Todavia, as fases de aleitamento prolongadas podem retardar a ingestão de alimentos sólidos, o que não foi observado neste trabalho, que poderia comprometer o desenvolvimento dos pré-estômagos dos cabritos, acarretando possível redução no crescimento do tamanho das papilas ruminais, que podem influenciar a absorção dos nutrientes e afetar seu desempenho após a fase de aleitamento (Lu \& Potchoiba, 1988).

Em criatórios com finalidade comercial, a utilização de leite de cabra para o aleitamento de cabritos pode ser uma prática antieconômica, pelo elevado valor do produto no mercado.

Desenvolvimento ponderal de cabritos após o aleitamento

Na Tabela 4, estão apresentados os resultados referentes ao desenvolvimento ponderal dos cabritos após as fases de aleitamento. Conforme as médias de peso observadas nas fases pós-aleitamento, verificou-se contínuo desenvolvimento para os animais, ocorrendo redução na diferença de ganho de peso entre os tratamentos, devido, possivelmente, a fatores climáticos, como fortes chuvas neste período, e problemas de ordem sanitária.

Os resultados obtidos para o tratamento 1 demonstraram a eficiência do desaleitamento de

Tabela 3 - Médias de pesos $(\mathrm{kg})$ por tratamentos e coeficiente de variação (CV) dos cabritos, de acordo com as fases de aleitamento

Table 3 - Means of weights $(\mathrm{kg})$ for treatments and coefficient of variation (CV) of kids, according to the suckling phases

\begin{tabular}{cccccr}
\hline $\begin{array}{c}\text { Idade (Dias) } \\
\text { Age (Days) }\end{array}$ & \multicolumn{3}{c}{$\begin{array}{c}\text { Tratamentos } \\
\text { Treatments }\end{array}$} & $\begin{array}{c}\text { Média } \\
\text { Mean }\end{array}$ & CV(\%) \\
\cline { 2 - 3 } & 1 & 2 & 3 & \\
\hline Nascimento & 2,6 & 3,3 & 2,7 & 2,8 & 15,2 \\
Birth & $4,5 \mathrm{a}$ & $4,6 \mathrm{a}$ & $4,0 \mathrm{~b}$ & 4,4 & 7,4 \\
14 & $6,0 \mathrm{a}$ & $6,1 \mathrm{a}$ & $5,4 \mathrm{~b}$ & 5,8 & 9,0 \\
28 & $8,4 \mathrm{ab}$ & $8,7 \mathrm{a}$ & $7,9 \mathrm{~b}$ & 8,3 & 9,3 \\
42 & $\mathbf{1 0 , 0 b}$ & $11,0 \mathrm{a}$ & $10,2 \mathrm{~b}$ & 10,4 & 9,1 \\
56 & $11,5 \mathrm{~b}$ & $\mathbf{1 3 , 0 a}$ & $12,6 \mathrm{a}$ & 12,4 & 10,4 \\
70 & $12,9 \mathrm{~b}$ & $14,7 \mathrm{a}$ & $\mathbf{1 4 , 9 a}$ & 14,1 & 11,0 \\
84 & & & & & \\
\hline
\end{tabular}

Médias seguidas de letras diferentes na mesma linha diferem entre si pelo teste de Duncan $(P<0,05)$. Means followed by different letters in the same row differ $(P<.05)$ by Duncan test.

Negrito = peso ao desmame

Bold = weaning weight .

R. Bras. Zootec., v.33, n.3, p.684-690, 2004 
cabritos a partir dos 56 dias de idade, acompanhado do fornecimento da dieta sólida a partir da segunda semana de vida, com redução na freqüência da dieta líquida após os 35 de vida, e do adequado manejo sanitário no cabriteiro e assepsia dos equipamentos; práticas que sugerem a adoção de criatórios de caprinos leiteiros, tornando a atividade mais prática e lucrativa, sem prejuízos no desempenho dos animais.

A média de peso vivo, obtida para os animais aos 98 dias de idade, foi de 15,6 kg, peso superior ao obtido por Medeiros (1996), que avaliou o efeito da freqüência do aleitamento sobre o desempenho de caprinos das raças Alpinas. Todavia, Susin (1990), estudando o aleitamento artificial de cabritos, encontrou resultados superiores aos relatados no presente estudo.

Os animais desaleitados aos 56 dias (T1) apresentaram peso médio aos 112 dias de 14,3 kg. Embora inferior aos dos tratamentos T2 e T3, foi superior aos encontrados por Santos et al. (1989) e Souza et al.(1992), avaliando o desempenho das raças Alpina e Anglo-Nubiana.

Ganho em peso de caprinos em diferentes fases de aleitamentos

A média de ganho de peso dos cabritos até os 56 dias de idade foi de $134 \mathrm{~g} / \mathrm{animal} /$ dia (Tabela 5), semelhante aos resultados encontrados por Medeiros (1996), porém, superior às médias observadas por Castro (1991) e Costa (1995) e inferior às obtidas por Susin (1990), utilizando leite de vaca e Agraz (1982), que obteve ganho médio de 157 g/animal/dia. Entretanto, deve-se considerar que esses últimos autores utilizaram quantidades elevadas de leite nos sistemas de aleitamento testados, aumentando, assim, os custos de produção.
Avaliando-se o ganho de peso dos cabritos dos 57 aos 70 dias de idade, constatou-se que não houve diferença significativa $(\mathrm{P}>0,05)$ para a média de ganho de peso, devido ao sexo dos animais. Todavia, verificou-se que, nos tratamentos 2 e 3 os cabritos ainda estavam submetidos à dieta líquida, o que favoreceu maior ganho de peso dos animais, quando comparados aos do tratamento 1.

Os resultados obtidos para os animais dos tratamentos 2 e 3, nos períodos de desmame, foram semelhantes aos observados por Geraseev (1995), em cabritos Saanen aleitados até os 91 dias de idade. Todavia, é necessária a análise do ganho após o aleitamento, para se verificar possibilidade de ganho compensatório, o que torna menos significantes as diferenças entre períodos de aleitamento, devendose considerar, ainda, o fator custo/benefício do leite em relação à carne.

No período de 85 a 182 dias de idade, verificou-se que não houve diferença significativa $(\mathrm{P}>0,05)$ do ganho de peso entre os tratamentos. Observou-se, também, que os machos apresentaram ganho de peso superior às fêmeas $(\mathrm{P}<0,05)$, resultados similares aos observados por Vélez-Nauer (1986) e Santos et al. (1989).

Apesar de os animais do tratamento 1 terem sido desaleitados mais precocemente, percebeu-se que, dos 85-112 dias de idade, os animais de todos os tratamentos apresentaram ganhos de peso semelhantes. Comportamento similar foi observado por MorandFehr et al. (1982), citado por Gouveia et al. (1998), comparando o desaleitamento de cabritos com quatro e seis semanas de idade, quando constataram o

Tabela 4 - Pesos médios (kg) e coeficiente de variação (CV) no período pós-aleitamento, em função dos tratamentos

Table 4 - Average weight $(\mathrm{kg})$ and coefficient of variation $(\mathrm{CV})$ during the powder-suckling period, according to each treatment

\begin{tabular}{|c|c|c|c|c|c|}
\hline \multirow{2}{*}{$\begin{array}{r}\text { Idade (dias) } \\
\text { Age (days) }\end{array}$} & \multicolumn{3}{|c|}{$\begin{array}{c}\text { Tratamentos } \\
\text { Treatments }\end{array}$} & \multirow{2}{*}{$\begin{array}{l}\text { Média } \\
\text { Average }\end{array}$} & \multirow[t]{2}{*}{ CV (\%) } \\
\hline & 1 & 2 & 3 & & \\
\hline 98 & $14,3 \mathrm{~b}$ & $15,8 a$ & $16,7 a$ & 15,6 & 12,0 \\
\hline 112 & $14,3 b$ & $16,4 \mathrm{a}$ & $17,5 \mathrm{a}$ & 16,1 & 12,3 \\
\hline 126 & $15,6 b$ & $17,1 \mathrm{ab}$ & 18,3а & 17,0 & 12,5 \\
\hline 140 & $16,3 b$ & 17,9ab & $19,0 a$ & 17,7 & 12,4 \\
\hline 154 & $16,7 b$ & $18,5 a$ & $19,5 a$ & 18,2 & 10,9 \\
\hline 168 & $18,0 \mathrm{~b}$ & $19,4 \mathrm{ab}$ & 20,9a & 19,4 & 11,3 \\
\hline 182 & $19,2 b$ & 20,3ab & $21,4 a$ & 20,3 & 10,6 \\
\hline
\end{tabular}

Médias seguidas de letras diferentes, na mesma linha, diferem $(P<0,05)$ entre si pelo teste Duncan. Means followed by different letters, in the same row, differ $(P<.05)$ by Duncan test.

R. Bras. Zootec., v.33, n.3, p.684-690, 2004 
Tabela 5 - Médias de ganho diário de peso dos cabritos (g) em diferentes fases, em função da duração do aleitamento e do sexo do animal

Table 5 - Averages of daily weight gain ( $g$ ) of kids at different phases, according to the suckling length and to animal sex

\begin{tabular}{|c|c|c|c|c|c|}
\hline \multirow[t]{2}{*}{$\begin{array}{c}\text { Idade (Dias) } \\
\text { Age (Days) }\end{array}$} & \multirow[t]{2}{*}{$\begin{array}{l}\text { Sexo } \\
\text { Sex }\end{array}$} & \multicolumn{3}{|c|}{$\begin{array}{l}\text { Tratamentos } \\
\text { Treatments }\end{array}$} & \multirow[t]{2}{*}{$\begin{array}{l}\text { Média } \\
\text { Average }\end{array}$} \\
\hline & & 1 & 2 & 3 & \\
\hline \multirow{3}{*}{$1-56$} & F & 129,0 & 129,0 & 132,0 & $130,0 \mathrm{~A}$ \\
\hline & M & 133,0 & 148,0 & 133,0 & $138,0 \mathrm{~A}$ \\
\hline & & $131,0 \mathrm{a}$ & 138,0a & $133,0 \mathrm{a}$ & 134,0 \\
\hline \multirow{3}{*}{$57-70$} & $\mathrm{~F}$ & 90,0 & 139,0 & 163,0 & $130,5 \mathrm{~A}$ \\
\hline & M & 142,0 & 136,0 & 189,0 & $155,5 \mathrm{~A}$ \\
\hline & & $114,0 \mathrm{~b}$ & 137,0ab & $175,0 \mathrm{a}$ & 142,0 \\
\hline \multirow{3}{*}{ 71-84 } & $\mathrm{F}$ & 73,0 & 98,0 & 169,0 & $113,0 \mathrm{~A}$ \\
\hline & M & 115,0 & 149,0 & 162,0 & $142,0 \mathrm{~A}$ \\
\hline & & $93,0 \mathrm{~b}$ & $122,0 \mathrm{~b}$ & $166,0 \mathrm{a}$ & 127,0 \\
\hline \multirow{3}{*}{ 85-182 } & $\mathrm{F}$ & 59,0 & 57,0 & 55,0 & $57,0 \mathrm{~B}$ \\
\hline & M & 73,0 & 57,0 & 78,0 & $69,0 \mathrm{~A}$ \\
\hline & & $65,0 a$ & $57,0 a$ & $66,0 \mathrm{a}$ & 62,5 \\
\hline \multirow{3}{*}{ 1-182 } & $\mathrm{F}$ & 84,0 & 89,0 & 96,0 & $89,6 \mathrm{~B}$ \\
\hline & M & 100,0 & 98,0 & 110,0 & $102,0 \mathrm{~A}$ \\
\hline & & $91,0 \mathrm{~b}$ & $93,0 \mathrm{~b}$ & $102,0 \mathrm{a}$ & 95,5 \\
\hline
\end{tabular}

Médias seguidas por diferente letra minúscula na mesma linha e diferente letra maiúscula na coluna, diferem $(\mathrm{P}<0,05)$ pelo teste de Duncan.

Means followed by different small letter in same row and different capital letters in the column, differ $(P<.05)$ by Duncan test.

mesmo ganho de peso nos dois grupos, ao analisarem os cinco primeiros meses. Perceberam, ainda, que os animais desaleitados com quatro semanas tiveram ganho inicial de peso menor; contudo, o crescimento compensatório reduziu este efeito. Da mesma forma, Louca et al. (1975) citaram que uma fase de aleitamento mais prolongada pode levar a ganho inicial em peso maior, todavia, a tendência é que haja posterior equilíbrio, devido ao ganho de peso compensatório. Porém, Battacharya (1980) encontrou melhores taxas de crescimento somente quando o consumo de leite foi elevado (dois litros/animal/dia).

Analisando-se a média geral no ganho em peso dos animais, do nascimento aos 182 dias de idade, verificou-se que os animais do tratamento 3 obtiveram ganho superior $(\mathrm{P}<0,05)$ aos animais dos tratamentos 1 e 2. Entretanto, é importante ressaltar que a superioridade do ganho de peso, para os animais desse tratamento, se deve ao fornecimento prolongado da dieta líquida, o que eleva os custos da produção deste sistema.

Foi constatado que o desaleitamento de cabritos aos 56 dias de idade demonstra viabilidade para o setor produtivo, proporcionando as seguintes vantagens: liberação de maior quantidade de leite para o mercado; disponibilidade de animais para a reposição e comercialização em menor espaço de tempo e redução na mão-de-obra, tornando o manejo do rebanho mais simples e econômico.

\section{Conclusões}

O desaleitamento aos 56 dias de idade proporcionou desenvolvimento satisfatório aos cabritos que, embora tenham revelado peso inferior aos desmamados mais tardiamente, apresentaram desempenho semelhante aos desaleitados aos 84 dias de idade.

Os animais submetidos ao desaleitamento aos 84 dias de idade apresentaram ganho de peso superior aos demais tratamentos, embora deva ser considerado o maior consumo de leite desses animais.

\section{Literatura Citada}

AGRAZ, A.A. Zôo-economics analysis of artificial and natural of kids. In: INTERNATIONAL CONFERENCE ON GOATS PRODUCTION AND DISEASE, 3., 1982, Tucson. Proceedings... Tucson: 1982. p.544.

ALVES, J.U. Cria de cabritos de raças leiteiras I. Desmame precoce. II. Aleitamento artificial. SIMPÓSIO NORDESTINO DE ALIMENTAÇÃO DE RUMINANTES, 4., 1992, Recife. Anais... Recife: 1992, p.91-107.

BARROS, N.N.; FERREIRA, M.P.B.; MELO, W.M.G. et al. Influência da dieta sobre o desempenho e o desenvolvimento 
do rúmen de cabritos na fase de aleitamento In: REUNIÃO ANUAL DA SOCIEDADE BRASILEIRA DE ZOOTECNIA, 35., 1998, Botucatu. Anais... Botucatu: Sociedade Brasileira de Zootecnia, 1998. p.263-265.

BHATTACHARYA, A.N. Research on goat nutrition and management in Mediterranean Middle East and Adjacent Arab Countries. Journal of Dairy Science, v.63, p.16811700, 1980.

CASTRO, I.M.C. Utilização de sucedâneos de leite no aleitamento de caprinos mestiços desmamados precocemente. Pirassununga: Universidade de São Paulo, 1991. 49p. Dissertação (Mestrado em Produção Animal) - Universidade de São Paulo, 1991.

COSTA, R.G.; RESENDE, K.T.; MARTINS, T.D.D. et al. Efeito do sistema de aleitamento no peso ao desmame de caprinos. In: REUNIÃO ANUAL DA SOCIEDADE BRASILEIRA DE ZOOTECNIA, 32., 1995, Brasília. Anais ... Brasília: Sociedade Brasileira de Zootecnia, 1995. p.176-178.

GERASEEV, L.C. Efeito do sistema de aleitamento sobre o desenvolvimento de caprinos. Jaboticabal: 1995, 47p. Trabalho de conclusão de curso (Graduação em Zootecnia). Universidade Estadual Paulista, 1995.

GOUVEIA, J.L.; QUEIROZ, A.C.; ESPESCHIT, C.J.B. et al. Efeito do manejo alimentar sobre a idade à desmama em caprinos leiteiros mestiços. In: REUNIÃO ANUAL DA SOCIEDADE BRASILEIRA DE ZOOTECNIA, 35., 1998. Botucatu. Anais... Botucatu: Sociedade Brasileira de Zootecnia, 1998. p.211.

LOUCA, A.; MAVROGENIS, A.; LAWLOR, M. J. The effect of early weaning on the lactation performance of Damascus goats and the growth rate of the kids. Animal Production, v.20, p.213-218, 1975.

LU, C.D.; POTCHOIBA, M.J. Nutrition and management of growing goats. In: ENCONTRO ANUAL SOBRE CAPRINOCULTURA DE LEITE, 4., 1988, Nova Odessa. Anais... Nova Odessa: Sociedade Brasileira de Zootecnia, 1988. p.61-77.

MAVROGENIS, A.P.; CONSTANTINOU, A.; LOUCA, A. Environmental and genetic causes of variation in production traits of Damascus goats. Animal Production, v.38, p.91-97, 1984.

MEDEIROS, A.N. Efeito do sistema de aleitamento sobre o desempenho de caprinos. Recife: Universidade Federal Rural de Pernambuco, 1996. 62p. Dissertação (Mestrado em Produção Animal) - Universidade Federal Rural de Pernambuco, 1996.

MORAND-FEHR, P.; HERVIEN, J.P.B.; SAUVANT, D. Feeding of young goats. INTERNATIONAL CONFERENCE OF GOATS PRODUCTION AND DISEASE, 3., 1982, Tucson. Proceedings ... Tucson, 1982. p.90-104.
MOUCHREK, E.; MOULIN, C.H.S. A importância do binômio freqüência x consumo diário no aleitamento artificial de caprinos. Informe Agropecuário, v.13, n.146, p.20-23, 1987.

MOUCHREK, E. Manejo de cabritos na fase de aleitamento. In: Caprinocultura e ovinocultura. João Pessoa: Sociedade Brasileira de Zootecnia, 1991. p.1-26.

NATIONAL RESEARCH COUNCIL - NRC. Nutrient requirements of goats: angora, dairy, and meat goats in temperate and tropical countries. Washington, D.C.: Nacional Academy of Science, 1981. p.91.

OWEN, E.; PAIVA, P. Artificial rearing of goat kids: effect of age at weaning and milk substitute restriction on performance to slaughter weight. Animal Production, v.30, n.480 (Alest.). p.270, 1980.

SANCHES, L.N. O aleitamento artificial dos cabritos. Boletim Informativo da Caprileite, n.8, v.43, p.13-17, 1985.

SANTOS, E.S.; RIBEIRO, N.M.; SANTOS, C.L.F. Aspectos genéticos e de meio sobre os pesos pré-desmama em caprinos de raças leiteiras. Pesquisa Agropecuária Brasileira, v.24, n.11, p.1301-1307, 1989.

STATISTICAL ANALYSES SYSTEM - SAS. User's guide: statistcs. Cary: 1992.

SILVA, L.R.F.; FIGUEREDO, P.A.E.; SIMPLÍCIO, A. A. et al. Parâmetros Genéticos e fenotípicos para os pesos de Caprinos Nativos e exóticos, criada no Nordeste do Brasil , na fase de crescimento. Revista da Sociedade Brasileira de Zootecnia, v.22, n.2, p.351-359, 1993.

SOUSA, W.H.; BARROS, N.N.; MOUCHREK, E. Sistemas alternativos de alimentação de cabritos desmamados precocemente. João Pessoa: EMEPA, 1992. 12p. (Boletim de Pesquisa, 3)

SUSIN, I. Manejo de caprinos jovens de raças leiteiras.In: SIMPÓSIO SOBRE PRODUÇÃO ANIMAL, 2., 1990, Campinas. Anais... Piracicaba: Fundação de Estudos Agrários Luiz de Queiroz, 1990. p.157-170.

UGIETTE, S.M.A. Utilização do soro de queijo no aleitamento artificial de cabritos. Areia: Universidade Federal de Paraíba, 1993. 83p. Dissertação (Mestrado em Produção Animal) - Universidade Federal de Paraíba, 1993.

VELEZ-NAUER, M. La crianza de cabras y ovejas en el trópico. ZAMORANO/WINROCK INTERNATIONAL, RTAC/EAP, 1986. p.261.

Recebido em: 12/11/01 Aceito em: 09/09/03 